Analytical and Bioanalytical Chemistry (2019) 411:1525-1536

https://doi.org/10.1007/s00216-019-01586-8

\title{
Development and application of water compatible molecularly imprinted polymers for the selective extraction of carbamazepine from environmental waters
}

\author{
Porkodi Kadhirvel ${ }^{1}$, Audrey Combes ${ }^{1}$, Louis Bordron ${ }^{1}$, Valerie Pichon 1,2 * \\ ${ }^{1}$ Dept of Analytical, Bioanalytical Sciences and Miniaturization (LSABM), CNRS, ESPCI Paris, \\ PSL Research University, 10 rue Vauquelin, 75231 Paris Cedex 05, France \\ ${ }^{2}$ Sorbonne Université, 4 Place Jussieu, 75005 Paris, France
}

Corresponding author: Valérie Pichon, valerie.pichon@espci.fr, +33140794772

Corresponding author institution: LSABM, ESPCI-Paris, 10 rue Vauquelin, 75005 Paris, France.

\begin{abstract}
A molecularly imprinted polymer (MIP) was designed in order to allow the selective solid-phase extraction of carbamazepine (CBZ), an anticonvulsant and mood-stabilizing drug, at ultra-trace level from aqueous environmental samples. A structural analog of CBZ was selected as a dummy template and different synthesis conditions were screened. The selectivity of the resulting imprinted polymers was evaluated by studying the retention of CBZ in a solvent similar to the one used for the synthesis. The presence of imprinted cavities in the polymers was then demonstrated by comparing the elution profiles (obtained by using MIP and a non-imprinted polymer, NIP, as a control) of the template, of CBZ, and of a structural analog of CBZ. Then the extraction procedure was further optimized for the treatment of aqueous samples on the two most promising MIPs, with a special attention being paid to the volume and composition of the percolation and washing solutions. The best MIP provided a highly selective retention in tap water with $81 \%$ extraction recovery for CBZ in the elution fraction of the MIP and only $14 \%$ for NIP. The repeatability of the extraction procedure was demonstrated for both tap and river waters (RSD below $4 \%$ in river water) for the drugs CBZ, oxcarbamazepine, and one metabolite (carbamazepine 10,11-epoxide). A MIP capacity of $1.15 \mu \mathrm{mol} \mathrm{g}^{-1}$ was determined. Finally, an analytical procedure involving the MIP was developed allowing the detection of CBZ at a concentration level of only a few $\mathrm{ng} \mathrm{L}^{-1}$ in river water. The selectivity provided by the MIP resulted in a 3000-fold increase of the signal-to-noise ratio in LC/MS analysis as compared to the use of conventional sorbent.
\end{abstract}

Keywords: Carbamazepine; Molecularly imprinted polymers; Solid phase extraction; Liquid chromatography; Mass spectrometry; Environmental aqueous samples

\section{Introduction}

Carbamazepine (CBZ) is an anticonvulsant and mood-stabilizing drug used worldwide for the treatment of temporal lobe epilepsy and neuropathic pain [1]. It is one of the most frequently detected pharmaceutical compounds in environmental waters $[2,3]$. After human consumption, up to $10 \%$ of CBZ is excreted unmetabolized from the body [3]. CBZ is resistant to biodegradation and with the relatively high level of excretion, the conventional wastewater treatment processes used by drinking water suppliers do not completely remove the CBZ from the waste water $[4,5]$. CBZ is therefore detected all over the world in ground, surface and waste water and even in 
drinking water at concentration levels ranging from a few $n g \mathrm{~L}^{-1}$ to several $\mu \mathrm{g} \mathrm{L}^{-1}$ [6]. At this low concentration, CBZ has no acute toxic effects in the aquatic ecosystem, however, potentially adverse effects can occur with continuous exposure and/or with the possible synergistic effects with other pollutants [7]. Different analytical methods have been developed for the quantification of CBZ by GC, LC and CE [8-10]. However, at this concentration level a sample treatment step by liquid-liquid extraction (LLE) or solid phase extraction (SPE) is necessary prior to analysis to allow a reliable quantitation of CBZ in aqueous samples [11-14].

Molecularly imprinted polymers (MIPs) have already been proposed for improving the selective trapping of CBZ [3, 15-24]. MIPs are material synthesized in the presence of a template molecule, leading to the formation of a polymer that possesses cavities that are complementary in size, shape, and the functionalities of the template compound. The MIP cavities have the ability to selectively retain target analytes from complex samples. The shape of the cavities, the nature of the monomer, and the porogen determine the nature and the strength of interactions that will occur between the target compounds and the imprinted polymer during the extraction procedures. Their stability under different $\mathrm{pH}$, solvent, or temperature conditions makes MIPs excellent sorbents for SPE compared with other selective sorbents such as immunosorbents [25]. In order to assess the selectivity of the retention observed on the MIP, a non-imprinted polymers (NIPs) are synthesized under the same conditions as MIPs, but without the introduction of the template molecule.

MIPs specific to CBZ have been prepared systematically using CBZ as a template molecule [3, 15-23]. In most cases, methacrylic acid (MAA) was used as a monomer [15-20, 22, 23] in association with ethylene glycol dimethacrylate (EGDMA) [20, 22, 23], trimethylolpropane trimethacrylate (TRIM) [18, 19] or with divinyl benzene (DVB) [15-17] as the cross-linker. Surprisingly, so far only three studies have reported on the screening of different synthesis conditions $[16,18,19]$. One study reported the effect of the ratio between MAA and methyl methacrylic acid (MMA) on the binding capacity and on the morphology of nanoparticles of a MIP synthesized with TRIM as the cross-linker and acetonitrile (ACN) as the porogen [19]. The same group later focused on the effect of the porogenic solvent (ACN, chloroform or toluene) in association with MAA and TRIM on the same parameters [18]. The third study only dealt with the effect of the cross-linker used (DVB or EGDMA) in association with MAA and a mixture of ACN and toluene as porogen on the extraction recovery of $\mathrm{CBZ}$ and on the selectivity of the resulting MIP [16].

MIPs have been applied to extract CBZ from pure media [18, 22], urine [15, 16, 23], serum [20], and plasma [23]. Only two studies have focused on the application of MIPs to extract CBZ from environmental aqueous samples $[15,17]$. In the first study, a high recovery yield $(80 \%)$ was reported for CBZ extracted from $100 \mathrm{~mL}$ of spiked waste water samples at a concentration level of $1 \mu \mathrm{g} \mathrm{L}^{-1}[15]$ using MIP, but no recovery yield was determined using the corresponding NIP. The actual retention selectivity of CBZ on the MIP in this matrix was then not demonstrated. In the second study, MIP was used for the removal of CBZ from real river water samples at concentration level of few $\mu \mathrm{g} \mathrm{L}^{-1}[17]$, but no extraction recoveries were described.

The aim of this work was thus to evaluate the potential of MIPs for the selective extraction of CBZ at ultra-trace levels (few ng $\mathrm{L}^{-1}$ ) from environmental samples. The MIP synthesis requires the introduction of a large quantity of the template molecule, and regarding the targeted concentrations in real environmental samples (highest contamination levels are of the order of a few $\mu \mathrm{g} \mathrm{L}^{-1}[6]$ ), Contrary to all previous studies an analog of CBZ was selected as the template molecule. Different 
conditions of synthesis were evaluated by changing the nature of the monomers, the cross-linker, and the porogen. The selection of the optimal synthesis conditions was achieved by studying the retention of the template, CBZ, and a structural analog of CBZ on each MIP and its corresponding NIP. After optimization of the extraction procedure, both in organic and aqueous medium, the MIP giving rise to the best selectivity for CBZ was studied in more detail by investigating its capacity and ability to extract CBZ from different water samples such as drinking, mineral, and river waters. To highlight the potential of the synthesized MIP, results obtained with the MIP were compared with results obtained by using a conventional Oasis HLB polymer for the analysis of a river water sample.

\section{Materials and methods}

\subsection{Materials}

Trifluoromethacrylic acid (TFMAA) was purchased from Apollo Scientific Ltd (Manchester, UK). Itaconic acid (IA), carbamazepine (CBZ), oxcarbamazepine (OX-CBZ) and iminostilbene (IMS) were procured from ABCR, Karlsruhe, Germany, while methoxycarbamazepine (MCBZ) came from Fluorochem Ltd (Derbyshire, UK), methacrylic acid (MAA), ethylene glycol dimethacrylate (EGDMA), divinylbenzene (DVB), triethanolamine (TEA) and carbamazepine 10,11-epoxide (CBZ-EPO) were from Sigma-Aldrich (Saint Quentin Fallavier, France). Washed EGDMA and MAA were distilled under vacuum in order to remove inhibitors. Azo-N,N'-bis-isobutyronitrile (AIBN) was purchased from Acros Organics (Noisy-le-Grand, France). HPLC-grade acetonitrile $(\mathrm{ACN})$, methanol $(\mathrm{MeOH})$, dichloromethane $(\mathrm{DCM})$, hexane and toluene were supplied by Carlo Erba (Val de Reuil, France). Acetic acid (AA) was purchased from VWR (Fontenay-sous-Bois, France). High purity water was dispensed by a Milli-Q purification system (Millipore, Saint Quentin en Yvelines, France).

\subsection{Instrumentation and Analytical conditions}

The LC-UV analyses were performed using a liquid chromatograph (LC) Agilent 1200 series system (Agilent Technology, Massy, France) equipped with a binary pump, an auto sampler and a diode array detector (DAD) controlled by Chemstation software. The chromatographic separation was performed on a Varian C18 column (Omnispher) $(150 \times 2.1 \mathrm{~mm}, 5 \mu \mathrm{m})$ maintained at $35^{\circ} \mathrm{C}$. The mobile phase, $\mathrm{MeOH} / \mathrm{ACN} / \mathrm{H}_{2} \mathrm{O}\left(38 / 20 / 42\right.$, v/v/v), flowed through the column at $0.2 \mathrm{~mL} \mathrm{~min}^{-}$ ${ }^{1}$ and the detection was performed by UV absorbance at $220 \mathrm{~nm}$. The injection volume was set at $5 \mu 1$. The corresponding calibration curves were furnished as supplementary material (Table S1).

The LC-MS/MS analyses were performed using a liquid chromatograph (UltiMate 3000®, Thermo Scientific, Illkirch, France) coupled with a Triple Stage Quadrupole Mass Spectrometer (TSQ Quantum Access MAX, Thermo Scientific, Illkirch, France) equipped with a heated electrospray ionization source (HESI2). The separation was performed under the same conditions as for LC/UV analysis. MS was operated in positive ion mode with MRM detection using an electrospray voltage of $3000 \mathrm{~V}$, a tube lens offset of 60 and $82 \mathrm{~V}$ for CBZ and MCBZ, respectively and 72V for CBZEPO and OX-CBZ. Capillary and vaporizer temperatures were set at $350{ }^{\circ} \mathrm{C}$ and $300{ }^{\circ} \mathrm{C}$ respectively. Nitrogen was used as desolvatation gas and argon as the collision gas at a pressure of 1.5 mTorr. Two transitions were monitored for each compound and collision energies were optimized and are indicated between parentheses: (i) CBZ: 237> 194 (19V), 237>179 (34V); (ii) MCBZ: 267> 222 (18V), 267> 193 (30V); (iii) CBZ-EPO : 253> 210 (14V), 253>180 (28V) and (iv) OX-CBZ : 253>236 (12V), 253>208 (19V). The corresponding calibration curves were furnished as supplementary material (Table S2). 
The instrument was also operated in full scan mode (Q3) $(\mathrm{m} / \mathrm{z}=100-1100)$ in order to visualize interfering compounds that were present in the elution fraction on the MIP/NIP and on a commercial Oasis HLB polymer (Waters).

\subsection{Synthesis of molecularly imprinted polymers and preparation of cartridges}

Five MIPs (MIP 1 to MIP 5) were synthesized by bulk polymerization using MCBZ as a template and different combinations of monomers, cross-linkers, and porogens. Non-imprinted polymers (NIP 1 to NIP 5), were synthesized following the same procedure as their corresponding MIP except that the template was absent. The five polymers were generated using a ratio $1 / 4 / 20$, the most common ratio used in the literature [25-31], between the template, the monomer, and the cross linker. For all the syntheses, the template $(1 \mathrm{mmol})$ and monomer $(4 \mathrm{mmol})$ were first mixed with $1.8 \mathrm{~mL}$ of the corresponding porogen in a glass vial the "template-monomer" complex was allowed to form in an ultra-sonication bath for 10 minutes. After this, $20 \mathrm{mmol}$ of the corresponding cross-linker was added and the mixture was purged for 10 minutes with a $\mathrm{N}_{2}$ stream to remove the dissolved oxygen and to avoid the quenching process. The thermal initiator, AIBN $(0.2 \mathrm{mmol})$, was then added to the above mixture and the glass vial was closed and placed in a $60^{\circ} \mathrm{C}$ water bath (this temperature was determined according to (i) the "monomer-template" complex that forms before the initiation of polymerization and that may be weakened by an excessively high temperature, (ii) the volatility of the porogen used and (iii) the necessity to promote the polymerization). After $48 \mathrm{~h}$ the glass vials were broken, and each polymer was crushed, ground automatically in a mixer (MIL MM 301 from Retsch ${ }^{\circledR}$ ) and sieved in a vibratory sieve shaker (Retsch ${ }^{\circledR}$ ). The particles between 25 and $36 \mu \mathrm{m}$ were collected and sedimented with $4 \mathrm{x} 5 \mathrm{~mL}$ of $\mathrm{MeOH} /$ water $(80 / 20, \mathrm{v} / \mathrm{v})$ to remove the thinest particles and then dried in a $40^{\circ} \mathrm{C}$ oven overnight.

Extraction cartridges were prepared by placing $55 \mathrm{mg}$ of the MIP or NIP particles between two polyethylene frits (Sigma-Aldrich) in $3 \mathrm{~mL}$ disposable polypropylene cartridges (Sigma Aldrich). The polymers were washed with $30 \mathrm{~mL}$ of $\mathrm{MeOH} / \mathrm{AA}(90 / 10, \mathrm{v} / \mathrm{v})$ to remove the template molecule. The washing fractions were evaporated under $\mathrm{N}_{2}$ and suspended in $\mathrm{MeOH} / \mathrm{ACN} / \mathrm{H}_{2} \mathrm{O}$ $(38 / 20 / 42, \mathrm{v} / \mathrm{v} / \mathrm{v})$ before injection into LC/UV. The washing step was continued until the template could no longer be detected in the washing fraction by LC/UV. Finally, the cartridges were washed with $10 \mathrm{~mL}$ of $\mathrm{MeOH}$ to remove residual AA. NIPs followed the same protocol.

\subsection{Evaluation of the MIPs in organic media}

For the screening of the different synthesis conditions and to assay the retention capacity and the selectivity in organic media only one extraction procedure was performed on each couple MIP/NIP. The cartridges were conditioned with $2.5 \mathrm{~mL}$ of a solvent similar to the one used as the porogen, i.e. toluene for MIP/NIP 1, MIP/NIP 4, and MIP/NIP 5 and DCM for MIP/NIP 2 and MIP/NIP 3. Then $1 \mathrm{~mL}$ of this solvent spiked with CBZ, MBZ, and IMS at a concentration level of $5 \mu \mathrm{g} \mathrm{mL}^{-1}$ was percolated through the MIP and the NIP. Elution was directly performed after percolation using $1 \mathrm{~mL}$ of a MeOH/AA, 90/10, v/v mixture. The elution fractions were evaporated under $\mathrm{N}_{2}$ stream and resuspended in $\mathrm{MeOH} / \mathrm{ACN} / \mathrm{H}_{2} \mathrm{O}(38 / 20 / 42$, v/v/v) before injection into LC/UV.

\subsection{Evaluation of the MIPs in aqueous media}

For extracting compounds from tap water, only one extraction procedure was performed on each couple MIP/NIP, the cartridges were conditioned with $2.5 \mathrm{~mL}$ of methanol and $2.5 \mathrm{~mL}$ of milliQ water before the percolation of $1 \mathrm{~mL}$ of tap water spiked at $5 \mu \mathrm{g} \mathrm{mL}^{-1}$ with CBZ, MBZ, and IMS. A washing step with $300 \mu \mathrm{L}$ of $0.1 \mathrm{M} \mathrm{HCl}$ followed by the same volume of milliQ water was added prior to the drying of the cartridge during 20 minutes under vacuum [26]. A second washing step 
adapted to each sorbent, was performed with $1 \mathrm{~mL}$ of toluene for MIP/NIP $1,1 \mathrm{~mL}$ of a mixture of DCM/hexane (30/70, v/v) for MIP/NIP 2 and MIP/NIP 3, $1 \mathrm{~mL}$ of DCM for MIP/NIP 4 and with $1 \mathrm{~mL}$ of a mixture DCM/toluene (25/75, v/v) for MIP/NIP 5. Finally the analytes were eluted with $1 \mathrm{~mL}$ of $\mathrm{MeOH} / \mathrm{AA}, 90 / 10, \mathrm{v} / \mathrm{v}$. The washing and elution fractions were evaporated under $\mathrm{N}_{2}$ stream and resuspended in $\mathrm{MeOH} / \mathrm{ACN} / \mathrm{H}_{2} \mathrm{O}(38 / 20 / 42$, v/v/v) before injection into LC/UV.

\subsection{Optimization of the extraction procedure in aqueous media}

For determining the breakthrough volumes, the cartridges were conditioned in the same way as for the extraction procedure described in Section 2.5. Different volumes ranging from $1 \mathrm{~mL}$ to $35 \mathrm{~mL}$ of tap water spiked with the same quantity (500 ng) of CBZ were passed through MIP 4 . All the other steps of the extraction procedure were performed as described in Section 2.5 for MIP 4 and the percolation fraction was lyophilized and resuspended in $\mathrm{MeOH} / \mathrm{ACN} / \mathrm{H} 2 \mathrm{O}(38 / 20 / 42$, v/v/v) before injection into LC/UV.

For the optimization of the washing conditions, different compositions, adapted to each MIP, with various polarities were tested. All the steps of the extraction procedure were the same as in section 2.5 except that $25 \mathrm{~mL}$ of tap water was percolated, followed by the second washing step of (i) 1.5 $\mathrm{mL}$ of DCM, or DCM/toluene $(80 / 20, \mathrm{v} / \mathrm{v})$, or DCM/hexane $(80 / 20, \mathrm{v} / \mathrm{v})$ or DCM/hexane $(70 / 30$, $\mathrm{v} / \mathrm{v}$ ) mixture for MIP/NIP 1 , and (ii) $1.5 \mathrm{~mL}$ of $\mathrm{DCM}$, or $\mathrm{DCM} /$ toluene $(50 / 50, \mathrm{v} / \mathrm{v}$ ), or DCM/hexane $(80 / 20, v / v)$ or DCM/hexane $(60 / 40, v / v)$ mixture for MIP/NIP 4. Finally the elution was performed with $1 \mathrm{~mL}$ of $\mathrm{MeOH}$. The elution fractions were evaporated under $\mathrm{N}_{2}$ stream and resuspended in $\mathrm{MeOH} / \mathrm{ACN} / \mathrm{H}_{2} \mathrm{O}(38 / 20 / 42$, v/v/v) before injection into LC/UV.

\subsection{Characterization by solid phase extraction of MIP 4 in different types of water}

To estimate the binding capacity of this MIP, $25 \mathrm{~mL}$ of tap water spiked with concentrations of CBZ ranging from $1 \mathrm{ng} \mathrm{mL} \mathrm{m}^{-1}$ to $1600 \mathrm{ng} \mathrm{mL}^{-1}$ were percolated through MIP 4 (corresponding to a quantity of CBZ between $25 \mathrm{ng}$ and $40 \mu \mathrm{g}$ ). The optimized extraction procedure was applied: a conditioning step with $2.5 \mathrm{~mL}$ of methanol and then $2.5 \mathrm{~mL}$ of milliQ water before the percolation of $25 \mathrm{~mL}$ of tap water spiked at different concentration with the targeted CBZ. A first washing step with $300 \mu \mathrm{L}$ of $0.1 \mathrm{M} \mathrm{HCl}$ followed by the same volume of milliQ water was performed prior to the drying of the cartridge for 20 minutes under vacuum. Then $1.5 \mathrm{~mL}$ of a mixture of DCM/hexane $(60 / 40, v / v)$ was used as the washing solution. Finally the analytes were eluted with $1 \mathrm{~mL}$ of $\mathrm{MeOH}$. The washing and elution fractions were evaporated under $\mathrm{N}_{2}$ stream and resuspended in $\mathrm{MeOH} / \mathrm{ACN} / \mathrm{H}_{2} \mathrm{O}(38 / 20 / 42, \mathrm{v} / \mathrm{v} / \mathrm{v})$ before injection into LC/UV and quantification thanks to calibration curves.

In order to check the repeatability of the extraction procedure and also the synthesis of the MIP, a new synthesis, named MIP 4-bis/NIP 4-bis, was carried out and the optimal extraction procedures were performed three times with tap water spiked at $20 \mathrm{ng} \mathrm{mL}^{-1}$.

Before applying Loire river water (collected near Orleans) onto the MIP/NIP 4 a double filtration was attempted using Millipore ${ }^{\circledR}$ glass-fiber filters (Merck, Ireland). For mineral (Evian water) and Loire river water, the optimal extraction procedure was applied in triplicate except that the spiking level was fixed at $500 \mathrm{ng} \mathrm{L}^{-1}$ of CBZ, MCBZ, CBZ-EPO, and OX-CBZ. This low concentration in these matrices necessitates the use of LC/MS-MS for the determination of the recovery yields in the fractions thanks to calibration curves. 


\subsection{Application of the optimized extraction procedure to different types of water}

To assess the selectivity of the MIP, a Loire river water sample was percolated through a conventional sorbent, an Oasis HLB polymer 3cc, $60 \mathrm{mg}$ (Waters) and on the M4-bis/N4-bis. The procedure for the MIP 4-bis/NIP 4-bis was exactly the same as those described in Section 2.7 except that the Loire river water was spiked at $100 \mathrm{ng} \mathrm{L}^{-1}$. For the Oasis sorbent, the extraction procedure was adapted from [32]: briefly the cartridge was conditioned with $2 \mathrm{~mL}$ of $\mathrm{MeOH}$ and $2 \mathrm{~mL}$ of water. After this conditioning step, $25 \mathrm{~mL}$ of the Loire river water spiked at $100 \mathrm{ng} \mathrm{L}^{-1}$ of CBZ were percolated through the cartridge, followed by $1 \mathrm{~mL}$ of water/MeOH $(90 / 10$, v/v) mixture as the washing step. The sorbent was dried for 20 min under vacuum before the elution of CBZ with $1.5 \mathrm{~mL}$ of $\mathrm{MeOH}$. The elution fractions collected from both sorbents (Oasis and MIP) were evaporated under an $\mathrm{N}_{2}$ stream and resuspended with $\mathrm{MeOH} / \mathrm{ACN} / \mathrm{H}_{2} \mathrm{O}(38 / 20 / 42$, v/v/v) before injection into LC/MS-MS. The elution fraction was injected twice, the first one in MRM mode for performing the quantification of CBZ and determining the recovery yield thanks to the LC/MSMS calibration curves, and the second time in scan mode $(\mathrm{m} / \mathrm{z}=100-1100)$ in order to visualize the cleanup effect of the extraction on Oasis HLB and on the MIP.

\section{Results and Discussions}

\subsection{Screening of the synthesis conditions}

\subsubsection{Choice of the synthesis conditions}

In order to avoid the risk of false positives caused by the leakage of CBZ when applying the MIP to real samples, a structural analog, the 10-methoxycarbamazepine (MCBZ) was selected as the template for the synthesis of all the MIPs in this study. MCBZ shares a tricyclic hydrophobic ring with the target compound (CBZ) and has a hydrophilic carboxamide group attached (Table 1).

Table 1: Chemical structure and partition coefficient of template, target and analog

$\begin{array}{ccc}\text { Chemical name Chemical structure } & \text { Log P } \\ \text { Carbamazepine } \\ \text { (CBZ) } \\ \text { Iminostilbene } \\ \text { (IMS) }\end{array}$


In order to promote hydrogen bond formation with the template, three polar and acidic functional monomers with different $\mathrm{pKa}$ values i.e. TFMAA ( $\mathrm{pKa}=2.1)$, MAA $(\mathrm{pKa}=4.65)$, and IA $(\mathrm{pKa}=$ 3.84 and 5.55) were studied (Table 2). These three monomers were evaluated in combination with two cross-linking agents, the polar and acidic EGDMA, and hydrophobic DVB that contains a phenyl group able to promote $\pi-\pi$ interactions (Table 2 ). DCM was initially chosen as the porogen for its non protic and moderate polar properties meaning that it favours the establishment of hydrogen bonds between the monomers and the template while ensuring the solubilization of the reagents. It was then used to prepare MIPs using EGDMA as the cross-linker (MIP 2 and MIP 3). However, the solubility of DVB was too low in this solvent. Therefore, when TFMAA or MAA were used as monomer in association with DVB (MIP 1 and MIP 4), DCM was mixed with toluene (non-polar solvent) in order to allow the complete solubilization of the mixture. IA was used for the synthesis of the MIP 5, but is barely soluble in non-polar solvents [33], so a ternary mixture of toluene with acetonitrile and TEA was required. NIP 1 to NIP 5 were synthesized following the same procedure to their corresponding MIP but without the template. Altogether, five sets of MIPs (MIP 1- MIP 5) and NIPs (NIP 1- NIP 5) were synthesized and $55 \mathrm{mg}$ of each sorbent were packed in $3 \mathrm{~mL}$ disposable cartridge to evaluate their retention properties.

Table 2: Composition of the polymerization solution used for MIPs and NIPs syntheses

\begin{tabular}{cccc} 
& Monomer & Cross linker & Porogen \\
\hline M1 & TFMAA & DVB & $\begin{array}{c}\text { Toluene/DCM, } \\
0.64 / 0.46, \mathrm{v} / \mathrm{v}\end{array}$ \\
\hline M2 & TFMAA & EGDMA & DCM \\
\hline M3 & MAA & EGDMA & DCM \\
\hline M4 & MAA & DVB & $\begin{array}{c}\text { Toluene/DCM, } \\
0.64 / 0.46, \mathrm{v} / \mathrm{v}\end{array}$ \\
\hline M5 & IA & DVB & $\begin{array}{c}\text { Toluene/ACN/TEA, } \\
0.47 / 0.47 / 0.6, \mathrm{v} / \mathrm{v} / \mathrm{v}\end{array}$ \\
\hline
\end{tabular}

\subsubsection{Retention of target analytes in organic media}

To evaluate the selectivity, i.e. the presence of cavities in MIPs, the retention of the template molecule (MCBZ) and of the target analyte (CBZ) was studied on the 5 couples of MIP/NIPs. In addition, IMS (no carboxamide group) (Table 1) was percolated to evaluate the contribution of the hydrogen bonds in the retention process. The initial screening studied the retention potential for these molecules, by percolating a pure organic media spiked with the three studied compounds. This solvent was selected to promote the same type of interaction that was developed during the imprinting process (toluene for MIP 1, MIP 4 and MIP 5, or DCM for MIP 2 and MIP 3). Indeed, if no selectivity is observed in this solvent, no selectivity can be expected in another solvent. In 
return, the direct application of a water with a not adapted washing procedure could give rise to a similar behavior on MIP and on NIP thus preventing the identification of the most promising sorbent. The elution step was performed with methanol acidified with $10 \%$ AA to disrupt polar interactions. The contribution of cavities in the retention process was evaluated by studying in parallel the retention on their corresponding NIPs.

The targeted CBZ (Figure 1A) and the template MCBZ (Figure 1B) exhibited the same extraction profiles regardless of the synthesized MIPs/NIPs. Since MCBZ has the same behavior as the target molecule during the extraction procedure, it definitively constitutes a good choice as the template molecule. MIP 1 and MIP 4 appear as the most promising MIPs in terms of retention and selectivity. These MIPs showed both a high recovery yield (81 and $95 \%$ for CBZ and $82 \%$ and $92 \%$ for MCBZ, respectively) in the elution fraction and a notable difference between the MIPs and the NIPs (only 12 and $67 \%$ of CBZ was retained until elution on NIP 1 and 4 respectively). DVB was used as the cross-linker in a mixture of toluene and DCM $(0.64 / 0.46, \mathrm{v} / \mathrm{v})$ as porogen and in association with two different acidic monomers TFMAA for MIP/NIP 1 and MAA for MIP/NIP 4, respectively. MIP 2 and MIP 3 were synthesized with the same acidic monomers (respectively TFMAA and MAA) as MIP 1 and MIP 4 respectively, but in association with another cross linker (EGDMA), and they all provided similar extraction profiles. Using DCM as the percolation solvent, the hydrogen bond formation was prevented between $\mathrm{CBZ}$ or MCBZ and the acidic monomer, leading to the loss of approximatively $80 \%$ of CBZ (or MCBZ) during the percolation step. The comparison of the profiles obtained on these four couples of MIP/NIPs highlight the role of DVB, used as a cross-linker in MIP 1 and MIP 4, in the retention of CBZ and MCBZ, probably because of $\pi-\pi$ interactions. This is confirmed by results obtained with MIP 5 that demonstrated a high retention of both compounds until elution (close to 100\%). However, as the profiles on NIP 5 were identical to those of MIP 5 for both molecules (Figure $1 \mathrm{~A}, \mathrm{~B}$ ), this retention is therefore not specific and is probably related: (i) to the use of IA that was not able to provide strong polar interactions in the solvent used for its solubilization, or (ii) according to the strong adsorption observed, the nonspecific binding interactions of IA functional groups cover up any template effects. Altogether, these results demonstrate that the nature of the monomer (acidic monomers) and of the cross-linker are crucial to promote retention and selectivity. The contribution of polar interactions (hydrogen bonds) in the retention mechanism is also confirmed by the extraction profiles obtained for IMS on all the synthesized MIP/NIPs. In the absence of the carboxamide moiety being able to establish these hydrogen bonds, IMS was not retained on any of the MIPs (Figure 1C), thus demonstrating the important role of the acidic monomer to ensure the retention of CBZ and MCBZ. The purpose of the study was to selectively extract CBZ from water, therefore it was necessary to study the ability of the different synthesized MIP/NIPs to develop specific interactions between the MIP and the targeted compounds in this aqueous media. 

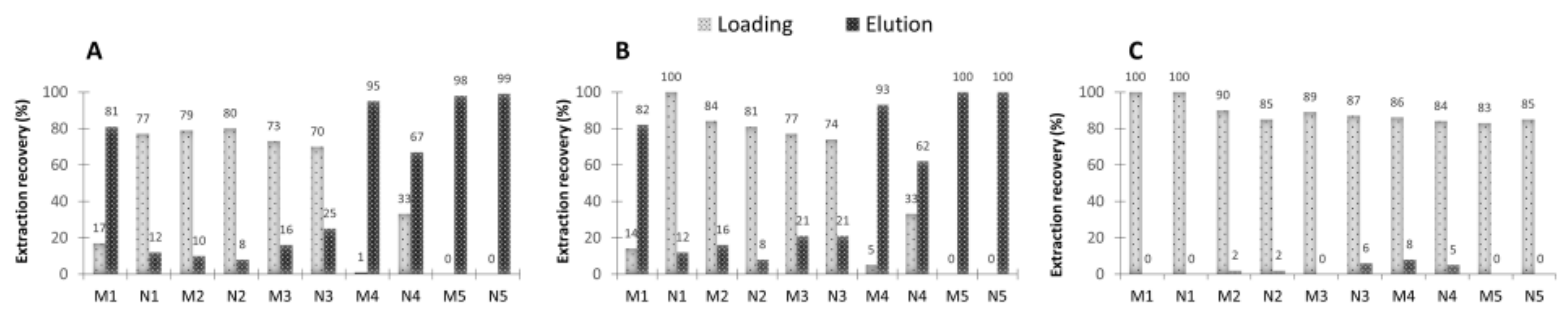

Figure 1: Extraction profiles obtained when loading (A) CBZ, (B) MCBZ and (C) IMS in organic media on the 5 synthesized MIPs and NIPs ( $\mathrm{n}=1$ ). Loading: $1 \mathrm{~mL}^{\text {of }} 5 \mathrm{mg} \mathrm{L}^{-1}$ in toluene (MIP 1, 4 et 5); in DCM (MIP 2 and 3); Elution : $1 \mathrm{~mL} \mathrm{MeOH/AA,} \mathrm{90/10,} \mathrm{v/v}$

\subsubsection{Evaluation of the MIPs in aqueous media}

During the percolation of water samples, the polarity of water prevents the establishment of hydrogen bonds, previously identified as essential to ensuring the selective retention of analytes on the MIP. Only hydrophobic non-specific interactions can be expected with the polymeric backbone. After the percolation step, a drying step was thus introduced to remove water and then to percolate a non-polar solvent, close to the porogen, as in section 3.1.2, to favor polar selective interactions. Different non-protic solvents with decreasing polarities were then tested as washing solution (DCM, toluene, or a mixture of DCM/toluene, or DCM/hexane) on the 5 synthesized MIP/NIPs. Moreover, in order to mimic the possible matrix effect that could occur during the analysis of real environmental samples, this study was performed with tap water. Environmental waters contain divalent ions that may cause ion-exchange between the protons of the carboxylic acid of the polymer. The divalent cations can replace the proton thus preventing the formation of hydrogen bonds necessary for selective retention on the MIP [26]. This matrix effect was circumvented by regenerating the interaction sites just before the drying step and the by washing with a $0.1 \mathrm{M}$ hydrochloric acid solution. The elution step was performed with $10 \%$ AA acidified methanol. The best extraction profiles i.e. those obtained with the washing solution that gives rise to a high recovery on the MIP and a low recovery on the NIP are presented in Figure 2 for CBZ, MCBZ and IMS.

MIP 2, MIP 3, and MIP 5 showed a high retention for both CBZ and MCBZ, but this was not selective since the retention was also strong on their corresponding NIPs. Both compounds were retained by non-specific interactions, in a range from 72 to $98 \%$ until elution without any significant difference between the MIPs and the NIPs. Conversely, MIP 1 and MIP 4 again showed a selective retention of CBZ and MCBZ (Figure 2A, B). Indeed, for these two MIPs, the recovery yield in the elution fraction, after washing with toluene for MIP 1 and DCM for MIP 4 (Figure 2A, B), was significantly higher on the MIP (for example, 96 and $100 \%$ for CBZ and MCBZ on MIP 1) than on the NIP ( 31 and $29 \%$ respectively). Under the same extraction conditions, IMS was partially or totally lost during the washing step for all the tested MIP/NIPs (Figure 2C) thus confirming the contribution of hydrogen bonds in the selective retention of CBZ and MCBZ. Interestingly, the extraction yield (55\%) of this compound in the elution fraction on MIP 1 demonstrated that the elution strength of the washing fraction had to be further optimized in order to remove possible interfering compounds with a polarity similar to IMS that could be retained by non-specific interactions. 

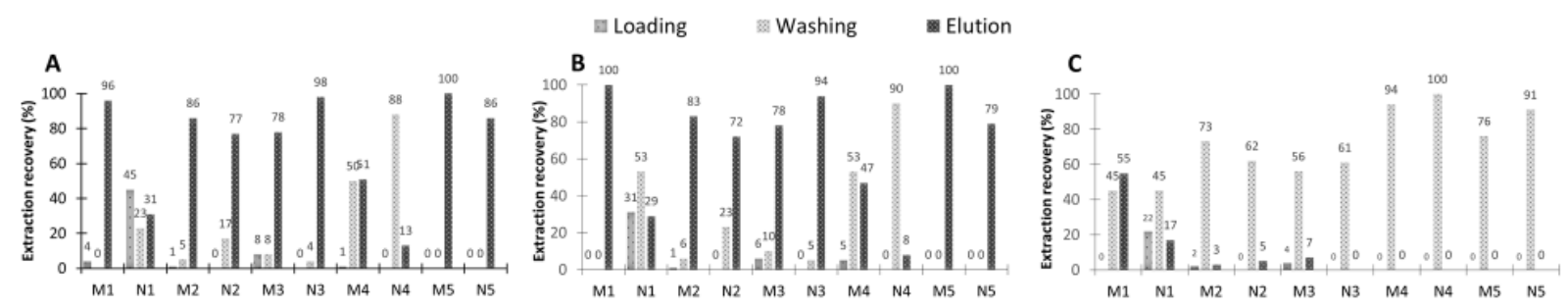

Figure 2: Extraction profiles obtained when percolating (A) CBZ, (B) MCBZ and (C) IMS in tap water on the 5 synthesized MIPs and NIPs $(n=1)$. Loading: $1 \mathrm{~mL}$ of $5 \mathrm{mg} \mathrm{L}^{-1}$ of each compound in tap water; washing: $1 \mathrm{~mL}$ of toluene for MIP/NIP $1,1 \mathrm{~mL}$ of DCM/hexane, 30/70, v/v, for MIP/NIP 2 and 3, $1 \mathrm{~mL}$ of DCM for MIP/NIP 4 and $1 \mathrm{~mL}$ of DCM/toluene, 25/75, v/v, for MIP/NIP; Elution: $1 \mathrm{~mL} \mathrm{MeOH/AA,} \mathrm{90/10,} \mathrm{v/v}$

Altogether, these results confirmed that MIP 1 and MIP 4 were the most promising in terms of the selective retention of CBZ. The further optimization of the extraction procedure was thus achieved only on these two imprinted sorbents and by limiting the study to CBZ and MCBZ.

\subsection{Optimization of the extraction procedure applied to real waters}

\subsubsection{Determination of the breakthrough volume}

The final aim of this work was to apply MIPs to the selective extraction of trace levels of CBZ from real environmental samples. Therefore, in order to obtain the highest possible enrichment factor and to decrease the quantification limits of the whole analytical procedure, increasing volumes of tap water $(15,25$, and $35 \mathrm{~mL}$ ) spiked with the same quantity of CBZ (500 ng), were percolated onto the MIPs and the corresponding extraction recovery yields in the percolation fraction were monitored. More than $93 \%$ of CBZ was retained on MIP 1 and MIP 4 during the percolation step with 15 and $25 \mathrm{~mL}$ of water. However, the percolation of $35 \mathrm{~mL}$ led to a decrease of the recovery on both sorbents thus indicating that the breakthrough volume was reached on both sorbents. In all the following experiments the loading volume was then fixed to $25 \mathrm{~mL}$ thus making it necessary to decrease the elution strength of the washing solution previously defined for the percolation of $1 \mathrm{~mL}$ of water samples.

\subsubsection{Optimization of the washing step}

The increase of the percolating volume up to $25 \mathrm{~mL}$ could lead to the migration of the target analytes through the sorbents during the percolation step and thus the elution strength of the solvent could be too high in the washing step. In order to avoid this possible effect, the washing step was optimized on MIP 1 and MIP 4 to decrease the retention on NIPs of the targeted CBZ (non-specific interactions at the surface of the polymer) while maintaining a high retention in the cavities of the MIPs. For that purpose, after the percolation of $25 \mathrm{~mL}$ of tap water spiked with CBZ and MCBZ, $1.5 \mathrm{~mL}$ of washing solutions of different elution strengths were assayed and the recovery in the elution fraction obtained with $1.5 \mathrm{~mL}$ of methanol were monitored. Pure methanol was then chosen because it elutes the target analytes with the same efficiency as the mixture MeOH/AA, 90/10, v/v and it greatly simplifies the conditioning step prior to the next analysis.

Different washing solutions $(1.5 \mathrm{~mL})$ including pure toluene, pure DCM and DCM containing various amounts of toluene $(20 \%)$ or hexane $(20 \%, 30 \%$ or $40 \%)$ were tested and the best compromise between retention and selectivity for MIP 1 was obtained using a DCM/Hexane mixture $(80 / 20, \mathrm{v} / \mathrm{v})$. This washing mixture allows for a high retention on the MIP $(87 \%$ of CBZ 
recovered in the elution fraction) while decreasing as much as possible the retention on the NIP (only $28 \%$ of CBZ retained until the elution fraction). For MCBZ, the recoveries were $53 \%$ and $13 \%$ in the elution fraction on the MIP and the NIP respectively. For MIP4/NIP4, the best compromise was obtained by applying a washing step with a mixture of DCM/hexane $(60 / 40, \mathrm{v} / \mathrm{v})$. Recovery in the elution fraction of the MIP were $75 \%$ and $88 \%$ for CBZ, and MCBZ and only $17 \%$ and $15 \%$, respectively, on the NIP. Thus, despite the fact that both MIPs were selective toward CBZ and MCBZ, the comparison of the results obtained in the best conditions defined for each couple of MIP/NIP showed that MIP 4 was more selective for the extraction of CBZ and MCBZ. The comparison of the selectivity can be achieved by comparing the ratios between the recoveries obtained on MIP and on NIP for a given molecule: these ratios were 3.07 and 4.07 for CBZ and MCBZ for MIP 1 and 4.41 and 5.86, respectively, for MIP 4. Thus, MIP/NIP 4 was selected for the remainder of the study.

\subsection{Capacity of the MIP}

The capacity corresponds to the maximum amount of a compound that can be retained by the imprinted polymer with a constant recovery. This depends directly on the number of cavities that are available in the polymer for the selective trapping of the target analytes. In order to evaluate this parameter in conditions as close as possible to the one used when applying the MIP4 to real samples, the evaluation of the capacity was carried out by percolating $25 \mathrm{~mL}$ of spiked drinking water and the optimized extraction procedure (washing with $1.5 \mathrm{~mL}$ of DCM/hexane, 60/40, v/v) was applied. The amounts of CBZ recovered in the elution fraction were plotted as a function of the percolated amount. As show in Figure 3, over the lowest concentrations studied, the trend was linear, meaning that the extraction recoveries remained constant from 0.02 to $0.6 \mu \mathrm{g} \mathrm{mL}^{-1}$. The slope of this linear part $(75 \%)$ corresponds to the constant recovery yield reflecting the selective retention of the analyte. For the highest concentrations of CBZ tested $\left(0.8\right.$ and $\left.1.6 \mu \mathrm{g} \mathrm{mL}^{-1}\right)$, the points were clearly not on the previous curve, meaning that the capacity of the cartridge was overloaded and that retention occurred because of non-specific interactions as observed with the NIP. The breakdown point of the curve, corresponding to the maximum amount of CBZ that can be retained on MIP with a constant recovery, was estimated to be close to $15 \mu \mathrm{g}$ of CBZ for $55 \mathrm{mg}$ of MIP, which corresponds to a capacity of $0.27 \mathrm{mg} \mathrm{g}^{-1}$ or $1.15 \mu \mathrm{mole}^{-1}$ of MIP. This value is in good agreement with those reported in the literature (between $0.2 \mu \mathrm{mol} \mathrm{g} \mathrm{g}^{-1}$ and $9 \mu \mathrm{mol} \mathrm{g}^{-1}$ ) for MIPs synthesized with the same combination of monomer (MAA) and cross linker (EGDMA) and targeted toward different classes of compounds with various physicochemical properties, like pesticides or drugs [29-33]. Moreover, the mean of the recoveries of $75 \pm 5 \%$ obtained for the extraction of CBZ from the water spiked between 0.02 and $0.6 \mu \mathrm{g} \mathrm{mL}^{-1}$ also highlights the reliability of the extraction procedure. 


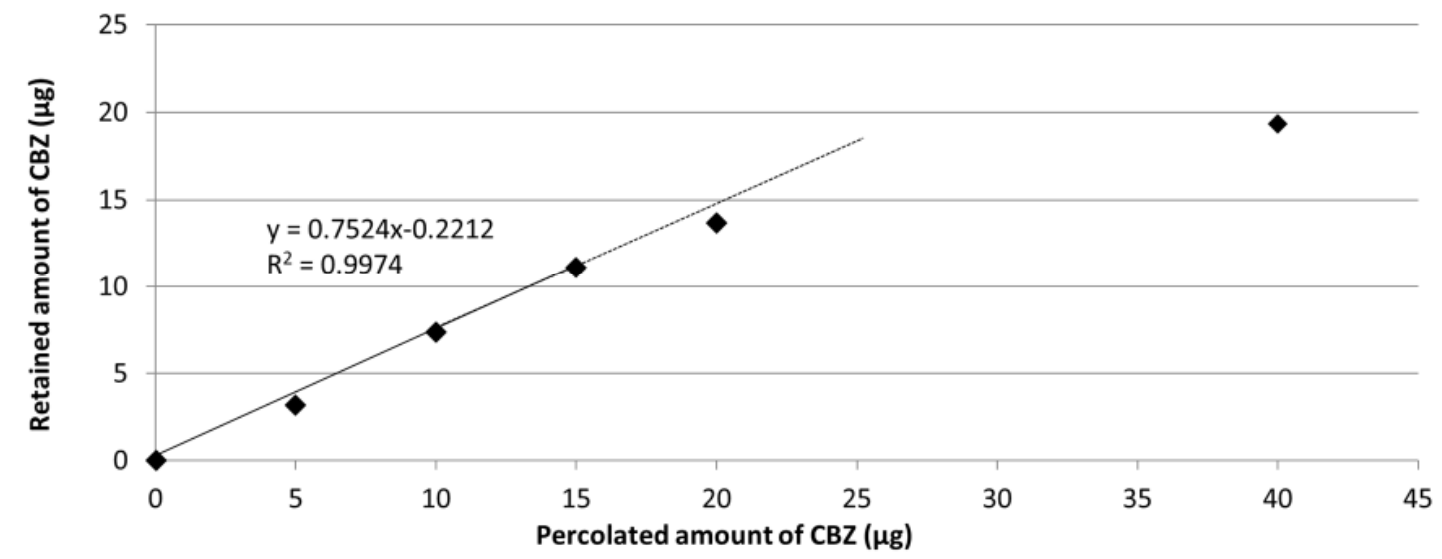

Figure 3: Capacity of MIP 4: Amount of CBZ ( $\mu$ g) quantified in the elution fraction obtained on MIP 4 as a function of the amount of CBZ percolated. The extraction procedure was composed of percolation: $25 \mathrm{~mL}$ of tap water spiked with various amount of CBZ; washing: $1.5 \mathrm{~mL}$ of DCM/hexane, $60 / 40, \mathrm{v} / \mathrm{v}$; Elution: 1 $\mathrm{mL} \mathrm{MeOH}$.

\subsection{Repeatability of the synthesis of the MIP}

To evaluate both the reliability of the synthesis of MIP 4 and the repeatability of the optimized extraction procedure on tap water, a new synthesis of MIP 4 was performed (named MIP 4-bis/NIP 4-bis) and three optimized extraction procedures were performed on M4-bis and on the corresponding N4-bis on three different days $(n=3)$. A high extraction recovery in the elution fraction for the targeted CBZ $(81 \pm 4 \%)$ and a high selectivity was again obtained using MIP 4 . The optimized extraction procedure led to a recovery of only $14 \pm 4 \%$ in the elution fraction on the NIP. The recovery yields for CBZ obtained on this MIP resulting from a second independent synthesis are in good agreement with those obtained on the first synthesized MIP 4/NIP 4 (75\% and $17 \%$ of recovery yield in the elution fraction of the MIP and of the NIP, respectively). Altogether these results demonstrate both the reliability of the synthesis and the repeatability of the developed extraction procedure.

\subsection{Repeatability of the optimized extraction procedure for different types of water}

The performance of this sorbent was then evaluated in two different aqueous media: (i) Evian water, commonly used by French water suppliers as model water to check their analytical procedure because of its saline and constant composition over time and (ii) Loire river water, a surface water directly taken from the river. The spiking level was lowered to $500 \mathrm{ng} \mathrm{L}^{-1}$, in order to be close to the level found in real media [6] and the SPE fractions were further analyzed by LC/MS-MS (mass spectrometry being selected for its higher sensitivity than UV).

The optimized extraction procedure was applied to an unspiked Loire river water sample and CBZ and MCBZ were detected at a concentration level of $80 \mathrm{ng} \mathrm{L}^{-1}$ and $1010 \mathrm{ng} \mathrm{L}^{-1}$, respectively (quantification performed thanks to LC/MS-MS calibration curves, supplementary materials 2). The extraction recovery for CBZ in spiked Loire river water was then calculated by taking into account the concentration detected $\left(80 \mathrm{ng} \mathrm{L}^{-1}\right)$ in an unspiked sample. Moreover, the use of the more sensitive MS detector (close to 1000 times more sensitive than UV) allowed for the detection of MCBZ in un-spiked samples which highlight the leakage of the template from the MIP (about $25 \mathrm{ng}$ of MCBZ were detected in the elution fraction). According to the quantity of MCBZ introduced during the synthesis for one cartridge $(0.2 \mathrm{mmol})$, this value corresponds to a leak at each extraction procedure of $0.000047 \%$ of the template molecule introduced during the synthesis. 
Even if this value remained really low, it clearly demonstrates the importance of the use of a structural analogue as a template during the synthesis process of a MIP in order to avoid false positive responses during its subsequent use, particularly when trace level concentrations are being investigated. The extraction recovery for MCBZ was then calculated for both Evian and Loire river water by taking into account the concentration detected $\left(1010 \mathrm{ng} \mathrm{L}^{-1}\right)$ in the un-spiked sample.

The extraction profile presented in Figure $4 \mathrm{~A}$ and $\mathrm{B}$ demonstrate that the selectivity of MIP 4 is maintained for the extraction of CBZ and MCBZ from Evian (Figure 4A) and Loire water (Figure 4B) with recoveries for CBZ of $70 \%$ and $65 \%$ on MIP 4 and only $31 \%$ and $20 \%$, respectively on the corresponding NIP. However the recovery yield decreased (70\% and $65 \%$, respectively, instead of $81 \%$ for tap water). The RSD values $(n=3)$ describing the repeatability of the extraction procedure for real samples were of $10 \%$ and $4 \%$ for Evian and Loire river water, respectively. These results clearly demonstrate the ability of the MIP to selectively extract CBZ from an environmental water sample at a concentration of only $500 \mathrm{ng} \mathrm{L}^{-1}$. However, one could wonder if, under the same conditions, MIP would be to trap simultaneously the two drugs CBZ and OX-CBZ and one metabolite (CBZ-EPO). Without any further optimization the full extraction procedure was applied and the extraction profiles obtained are presented in Figures 4A and B. MIP 4 was able to selectively extract these two compounds from the different types of water (extraction recovery in the elution fraction of $69 \%$ and $60 \%$ for CBZ-EPO and of $91 \%$ and $69 \%$ for OX-CBZ in Evian and Loire river water, respectively).

The reusability of imprinted polymers is crucial in order to provide a reliable, economic and sustainable sample treatment step. In this study, multiple extraction procedures were carried out using the same cartridge. Interestingly, even after 100 cycles (adsorption - elution), the MIP demonstrated no carry over (demonstrated thanks to the percolation of unspiked milliQ water, data not shown) and was able to selectively trap the CBZ in complex environmental samples with the same performance.
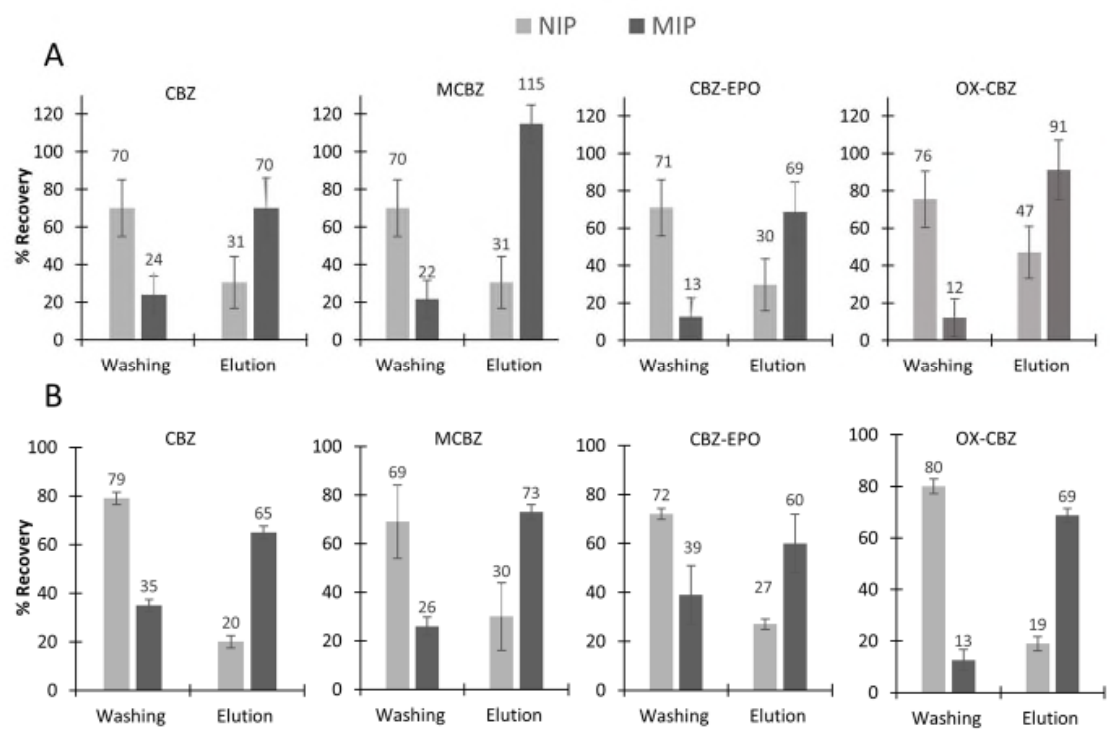

Figure 4: Repeatability of the extraction procedure $(n=3)$ obtained when percolating a mixture of CBZ, MCBZ, CBZ-EPO and OX-CBZ in (A) Evian water and (B) Loire river water on the MIP 4/NIP 4. The extraction procedure was the same as in Figure 3 except that the Evian and Loire river water were spiked at a concentration level for $500 \mathrm{ng} \mathrm{L}^{-1}$ of each compounds and analysis of the fractions were carried out in LC/MS-MS. 


\subsection{Comparison of performances with a conventional sorbent}

To highlight the potential of the MIP, its performances in terms of recovery yield or cleaning capacity was compared to those obtained with Oasis HLB sorbent which is commonly applied to the extraction of drugs from waters [32]. For this purpose, a sample of Loire river water spiked at $100 \mathrm{ng} \mathrm{L}^{-1}$ was extracted on both sorbents. The use of Oasis HLB led to a recovery yield of $74 \pm$ $6 \%$ in the elution fraction. This value was slightly higher than those obtained with the MIP (65 \pm 4\%). However, the comparison of the LC/MS chromatogram in scan mode (Figure 5) clearly showed that the elution fraction resulting from the Oasis HLB contained more interfering compounds (higher baseline signal, presence of many peaks and higher noise) than the elution fraction of the MIP. The presence of these interfering compounds raises the possibility that a matrix effect occurred during the LC/MS-MS analysis after an extraction on Oasis HLB which could induce an overestimation of the extraction recovery yield. In addition, the chromatogram in MRM mode (Figure 5, insert a and b) showed that, even in this mode with some selectivity, the signal to noise ratio corresponding to the quantitation ion of CBZ in the elution fraction of MIP (Figure 5, insert b) was 3000 times higher than in the same fraction of Oasis HLB (figure 5, insert a). This cleaner elution extract obtained using the MIP should provide an increase in the life time of the analytical column and limit the clogging of the source of the mass spectrometer. However the main advantage of using MIP was an important improvement in the limit of quantification of the global analytical procedure (sample treatment on MIP 4 and LC/MS-MS analysis). The high S/N observed fin the MRM mode for the Loire river water clearly demonstrated that sample treatment on MIP 4 coupled with LC/MS-MS analysis allows for the quantification of CBZ at a concentration level of only $4 \mathrm{ng} \mathrm{\textrm {L } ^ { - 1 }}$. CBZ was detected in surface water, waste water, and even in drinking water at concentrations that ranged from a few $\mathrm{ng} \mathrm{L}^{-1}$ to several $\mu \mathrm{g} \mathrm{\textrm {L } ^ { - 1 }}$ [6]. Thus, the developed MIP clearly demonstrates its potential for its application to environmental waters.

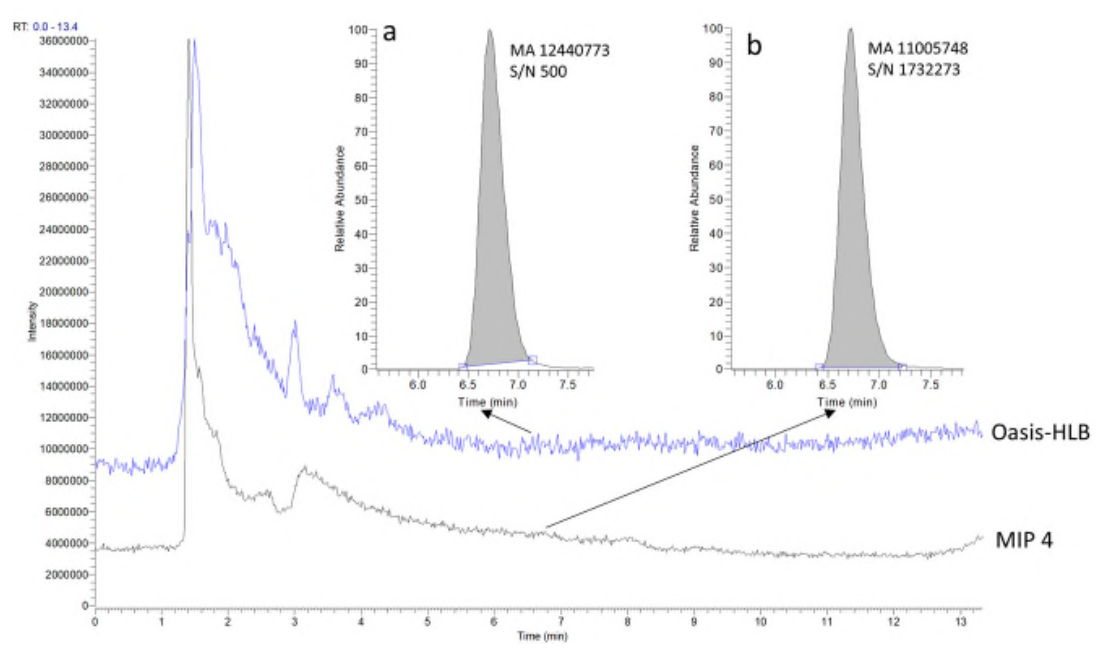

Figure 5: LC/MS chromatogram in scan mode $(\mathrm{m} / \mathrm{z}=100-1100)$ of the elution fraction obtained after the percolation of Loire river water on Oasis HLB and on MIP 4-bis. In the insert, the MRM chromatogram of the same samples corresponding to the quantitation transition for CBZ $(237 \rightarrow 194)$ in the elution fraction of Oasis HLB (a) and MIP 4 (b). The extraction procedure was the same as the one described in Figure 3, except that the Loire river water was spiked at a concentration level of $100 \mathrm{ng} \mathrm{L}^{-1}$ and analysis of the fractions was carried out in LC/MS-MS. 


\section{Conclusions}

Different conditions of synthesis were screened to determine the optimal conditions for the synthesis of a MIP able to selectively extract CBZ from pure organic and aqueous media. The most promising MIP was synthesized by using MCBZ as the template, MAA as the monomer, DVB as the cross linker, and a mixture of toluene and DCM as porogen. After studying the repeatability of the optimized SPE procedure and the reliability of the MIP synthesis in pure media, the capacity of the MIP for the trapping of CBZ was determined (1.15 $\mu$ mole $\mathrm{g}^{-1}$ of MIP) and the performances of this polymer was evaluated in real media. A high retention of $\mathrm{CBZ}$ was maintained with mineral and river water with extraction recoveries of 70 and $65 \%$ respectively, while the recoveries with the NIP were lower than $20 \%$. The MIP was also able to selectively trap selectively two other structurally similar compounds, another drug (OX-CBZ) and a metabolite (CBZ-EPO), under the same conditions. The repeatability of the extraction procedure was also demonstrated in these real media (RSD of 4\%). The MIP demonstrated an efficient clean-up of the sample compared with a conventional Oasis HLB sorbent, thus allowing for the quantification of CBZ by LC/MS-MS at the ultra-trace level $\left(4 \mathrm{ng} \mathrm{L}^{-1}\right)$ in Loire river water that is fully in accordance with the targeted concentration in real water samples. This work also highlighted the advantage of using a dummy molecule as the template to circumvent the risk of false positives.

\section{Acknowledgments:}

This work was supported by the French National Research Agency (ANR Program: ANR-15CE04-0012, project MIP_WQT).

\section{Compliance with ethical standards}

This is not a clinical study on humans/animals.

\section{Conflict of interest}

The authors declare that they have no conflict of interest.

\section{References:}

1. Heberer T, Feldmann D (2005) Contribution of effluents from hospitals and private households to the total loads of diclofenac and carbamazepine in municipal sewage effluents--modeling versus measurements. J Hazard Mater 122:211-218 . doi: 10.1016/j.jhazmat.2005.03.007

2. Ferrer I, Thurman EM (2012) Analysis of 100 pharmaceuticals and their degradates in water samples by liquid chromatography/quadrupole time-of-flight mass spectrometry. J Chromatogr A 1259:148157 . doi: 10.1016/j.chroma.2012.03.059

3. Luo Y, Guo W, Ngo HH, Nghiem LD, Hai FI, Zhang J, Liang S, Wang XC (2014) A review on the occurrence of micropollutants in the aquatic environment and their fate and removal during wastewater treatment. Sci Total Environ 473-474:619-641 . doi: 10.1016/j.scitotenv.2013.12.065

4. Wick A, Fink G, Joss A, Siegrist H, Ternes TA (2009) Fate of beta blockers and psycho-active drugs in conventional wastewater treatment. Water Res 43:1060-1074 . doi: 10.1016/j.watres.2008.11.031

5. Alvarino T, Suarez S, Lema JM, Omil F (2014) Understanding the removal mechanisms of PPCPs and the influence of main technological parameters in anaerobic UASB and aerobic CAS reactors. J Hazard Mater 278:506-513 . doi: 10.1016/j.jhazmat.2014.06.031 
6. Hai FI, Yang S, Asif MB, Sencadas V, Shawkat S, Sanderson-Smith M, Gorman J, Xu Z-Q, Yamamoto K (2018) Carbamazepine as a Possible Anthropogenic Marker in Water: Occurrences, Toxicological Effects, Regulations and Removal by Wastewater Treatment Technologies. Water 10:107 . doi: 10.3390/w10020107

7. Jos A, Repetto G, Rios JC, Hazen MJ, Molero ML, del Peso A, Salguero M, Fernández-Freire P, Pérez-Martín JM, Cameán A (2003) Ecotoxicological evaluation of carbamazepine using six different model systems with eighteen endpoints. Toxicol Vitro Int J Publ Assoc BIBRA 17:525-532

8. Huerta B, Jakimska A, Gros M, Rodríguez-Mozaz S, Barceló D (2013) Analysis of multi-class pharmaceuticals in fish tissues by ultra-high-performance liquid chromatography tandem mass spectrometry. J Chromatogr A 1288:63-72 . doi: 10.1016/j.chroma.2013.03.001

9. Lin Y-Y, Wang C-C, Ho Y-H, Chen C-S, Wu S-M (2013) Analysis of carbamazepine and its five metabolites in serum by large-volume sample stacking-sweeping capillary electrophoresis. Anal Bioanal Chem 405:259-266 . doi: 10.1007/s00216-012-6481-x

10. Durán-Alvarez JC, Becerril-Bravo E, Castro VS, Jiménez B, Gibson R (2009) The analysis of a group of acidic pharmaceuticals, carbamazepine, and potential endocrine disrupting compounds in wastewater irrigated soils by gas chromatography-mass spectrometry. Talanta 78:1159-1166 . doi: 10.1016/j.talanta.2009.01.035

11. Wad N (1984) Simultaneous determination of eleven antiepileptic compounds in serum by highperformance liquid chromatography. J Chromatogr 305:127-133

12. Gómez MJ, Petrović M, Fernández-Alba AR, Barceló D (2006) Determination of pharmaceuticals of various therapeutic classes by solid-phase extraction and liquid chromatography-tandem mass spectrometry analysis in hospital effluent wastewaters. J Chromatogr A 1114:224-233 . doi: 10.1016/j.chroma.2006.02.038

13. Franceschi L, Furlanut M (2005) A simple method to monitor plasma concentrations of oxcarbazepine, carbamazepine, their main metabolites and lamotrigine in epileptic patients. Pharmacol Res 51:297302 . doi: 10.1016/j.phrs.2004.09.008

14. Lajeunesse A, Vernouillet G, Eullaffroy P, Gagnon C, Juneau P, Sauvé S (2009) Determination of carbamazepine in aquatic organisms by liquid-liquid extraction and liquid chromatography-tandem mass spectrometry. J Environ Monit JEM 11:723-725 . doi: 10.1039/b820302b

15. Beltran A, Caro E, Marcé RM, Cormack P a. G, Sherrington DC, Borrull F (2007) Synthesis and application of a carbamazepine-imprinted polymer for solid-phase extraction from urine and wastewater. Anal Chim Acta 597:6-11 . doi: 10.1016/j.aca.2007.06.040

16. Beltran A, Marcé RM, Cormack P a. G, Borrull F (2009) Synthesis by precipitation polymerisation of molecularly imprinted polymer microspheres for the selective extraction of carbamazepine and oxcarbazepine from human urine. J Chromatogr A 1216:2248-2253 . doi: 10.1016/j.chroma.2009.01.024

17. Dai C, Geissen S-U, Zhang Y, Zhang Y, Zhou X (2010) Performance evaluation and application of molecularly imprinted polymer for separation of carbamazepine in aqueous solution. J Hazard Mater 184:156-163 . doi: 10.1016/j.jhazmat.2010.08.018

18. Esfandyari-Manesh M, Javanbakht M, Atyabi F, Badiei A, Dinarvand R (2011) Effect of porogenic solvent on the morphology, recognition and release properties of carbamazepine-molecularly imprinted polymer nanospheres. J Appl Polym Sci 121:1118-1126 . doi: 10.1002/app.33812

19. Esfandyari-Manesh M, Javanbakht M, Atyabi F, Dinarvand R (2012) Synthesis and evaluation of uniformly sized carbamazepine-imprinted microspheres and nanospheres prepared with different mole ratios of methacrylic acid to methyl methacrylate for analytical and biomedical applications. J Appl Polym Sci 125:1804-1813 . doi: 10.1002/app.36288

20. Esfandyari-Manesh M, Javanbakht M, Dinarvand R, Atyabi F (2012) Molecularly imprinted nanoparticles prepared by miniemulsion polymerization as selective receptors and new carriers for the sustained release of carbamazepine. J Mater Sci Mater Med 23:963-972 . doi: 10.1007/s10856-0124565-y 
21. Dai C, Zhang J, Zhang Y, Zhou X, Duan Y, Liu S (2013) Removal of carbamazepine and clofibric acid from water using double templates-molecularly imprinted polymers. Environ Sci Pollut Res Int 20:5492-5501 . doi: 10.1007/s11356-013-1565-5

22. Lee J-C, Kim C-R, Byun H-S (2014) Synthesis and adsorption properties of carbamazepine imprinted polymer by dispersion polymerization in supercritical carbon dioxide. Korean J Chem Eng 31:22662273 . doi: 10.1007/s11814-014-0178-0

23. Khalilian F, Ahmadian S (2016) Molecularly imprinted polymer on a SiO2 -coated graphene oxide surface for the fast and selective dispersive solid-phase extraction of Carbamazepine from biological samples. J Sep Sci 39:1500-1508 . doi: 10.1002/jssc.201501392

24. Zhang Y-L, Zhang J, Dai C-M, Zhou X-F, Liu S-G (2013) Sorption of carbamazepine from water by magnetic molecularly imprinted polymers based on chitosan-Fe3O4. Carbohydr Polym 97:809-816 . doi: 10.1016/j.carbpol.2013.05.072

25. Pichon V (2007) Selective sample treatment using molecularly imprinted polymers. J Chromatogr A 1152:41-53 . doi: 10.1016/j.chroma.2007.02.109

26. Chapuis F, Pichon V, Lanza F, Sellergren S, Hennion M-C (2003) Optimization of the class-selective extraction of triazines from aqueous samples using a molecularly imprinted polymer by a comprehensive approach of the retention mechanism. J Chromatogr A 999:23-33 . doi: 10.1016/S0021-9673(03)00552-1

27. Chapuis-Hugon F, Cruz-Vera M, Savane R, Ali WH, Valcarcel M, Deveaux M, Pichon V (2009) Selective sample pretreatment by molecularly imprinted polymer for the determination of LSD in biological fluids. J Sep Sci 32:3301-3309 . doi: 10.1002/jssc.200900247

28. Lasáková M, Thiébaut D, Jandera P, Pichon V (2009) Molecularly imprinted polymer for solid-phase extraction of ephedrine and analogs from human plasma. J Sep Sci 32:1036-1042 . doi: $10.1002 /$ jssc. 200800684

29. Duy SV, Lefebvre-Tournier I, Pichon V, Hugon-Chapuis F, Puy J-Y, Périgaud C (2009) Molecularly imprinted polymer for analysis of zidovudine and stavudine in human serum by liquid chromatography-mass spectrometry. J Chromatogr B Analyt Technol Biomed Life Sci 877:11011108 . doi: 10.1016/j.jchromb.2009.02.068

30. Thibert V, Legeay P, Chapuis-Hugon F, Pichon V (2012) Synthesis and characterization of molecularly imprinted polymers for the selective extraction of cocaine and its metabolite benzoylecgonine from hair extract before LC-MS analysis. Talanta 88:412-419 . doi: 10.1016/j.talanta.2011.11.009

31. Boulanouar S, Combès A, Mezzache S, Pichon V (2017) Synthesis and application of molecularly imprinted polymers for the selective extraction of organophosphorus pesticides from vegetable oils. J Chromatogr A 1513:59-68 . doi: 10.1016/j.chroma.2017.07.067

32. Ollers S, Singer HP, Fässler P, Müller SR (2001) Simultaneous quantification of neutral and acidic pharmaceuticals and pesticides at the low-ng/l level in surface and waste water. J Chromatogr A 911:225-234

33. Yang W, Hu Y, Chen Z, Jiang X, Wang J, Wang R (2012) Solubility of itaconic acid in different organic solvents: Experimental measurement and thermodynamic modeling. Fluid Phase Equilibria 314:180-184 . doi: 10.1016/j.fluid.2011.09.027 